\title{
Hysteretic Response of an Anti-Ferromagnetic Random-Field Ising Model in One Dimension at Zero Temperature
}

\author{
Prabodh Shukla, Ratnadeep Roy, and Emilia Ray \\ Physics Department \\ North Eastern Hill University \\ Shillong-793 022, INDIA
}

\begin{abstract}
We consider the hysteretic response of a one-dimensional anti-ferromagnetic random-field Ising model at zero temperature for a uniform bounded distribution of quenched random fields, and present analytic results in a limited range of the applied field.
\end{abstract}

email:shukla@dte.vsnl.net.in 


\section{INTRODUCTION}

Relaxation dynamics of random field Ising model (RFIM) at zero temperature provides a simple caricature of complex non-equilibrium phenomena. RFIM with ferromagnetic as well as anti-ferromagnetic interactions has been investigated in the context of two distinct classes of relaxational behavior. The ferromagnetic RFIM shows relaxation by avalanches and has been applied [1, 2] to study hysteresis and Barkhausen jumps in magnetic materials. The anti-ferromagnetic model does not support avalanches, and is more appropriate [3], (t] for the glassy kind of dynamics where relaxation may proceed by single localized events. In spite of the simplicity of RFIM and its relaxational dynamics at zero temperature, exact solution of the model is difficult on account of its randomness. So far, analytic solution of the model has been obtained in one dimension [5] and on a Bethe lattice [2, [6] for ferromagnetic interactions only. In this paper, we present a solution of the non-equilibrium dynamics of the anti-ferromagnetic RFIM at zero temperature in one dimension in a limited range of the applied field. For simplicity, we limit ourselves to a bounded uniform distribution of the random field. It is possible to extend this solution to other distributions of the random field, but this will not be taken up in this paper. 


\section{THE MODEL}

At each site of a one dimensional lattice, there is an Ising spin $s_{i}= \pm 1$, $i=1,2,3 \ldots \ldots, n$ which interacts with its nearest neighbors through an antiferromagnetic interaction J. Each site has a quenched random field $h_{i}$ drawn independently from a continuous bounded distribution,

$$
\begin{aligned}
p\left(h_{i}\right) & =\frac{1}{2 \Delta} & & \text { if } \quad-\Delta \leq h_{i} \leq \Delta \\
& =0, & & \text { otherwise. }
\end{aligned}
$$

The entire system is placed in an externally applied uniform field $h_{a}$. The Hamiltonian of the system is given by

$$
H=-J \sum_{i} s_{i} s_{i+1}-\sum_{i} h_{i} s_{i}-h_{a} \sum s_{i}
$$

We consider the hysteretic response of this system when the external field $h_{a}$ is slowly increased from $-\infty$ to $+\infty$. We assume the dynamics to be the single-spin-flip Glauber dynamics at zero temperature, i.e. a spin is flipped only if it lowers the energy. We assume that if the spin-flip is allowed, it occurs with a rate $\Gamma$, which is much larger than the rate at which the magnetic field $h_{a}$ is increased. Thus we assume that all flipable spins relax instantly, and the spin $s_{i}$ always has the same sign as the net local field $l_{i}$ at the site.

$$
s_{i}=\operatorname{sign} l_{i}=\operatorname{sign}\left[J\left(s_{i-1}+s_{i+1}\right)+h_{i}+h_{a}\right]
$$

The hysteretic response of the system to an applied field $h_{a}$ is measured by the magnetization $m\left(h_{a}\right)$ per spin, 


$$
m\left(h_{a}\right)=\frac{1}{N} \sum_{i} s_{i}
$$

We start with $h_{a}=-\infty$, when $m=-1$, and increase $h_{a}$ slowly to $h_{a}=+\infty$ when $m=1$. Our object is to calculate $m\left(h_{a}\right)$ for all values of $h_{a}$. The magnetization $m_{R}\left(h_{a}\right)$ on the return trajectory when $h_{a}$ is slowly decreased from $h_{a}=+\infty$ to $h_{a}=-\infty$ can be obtained from $m\left(h_{a}\right)$ by a symmetry relation $m_{R}\left(h_{a}\right)=-m\left(-h_{a}\right)$. Therefore the knowledge of the magnetization curve on the lower half of the hysteresis loop suffices to determine the entire hysteresis loop, and we can limit ourselves to the calculation of $m\left(h_{a}\right)$ alone.

\section{SIMULATIONS}

It is instructive to look at a computer simulation of the model before proceeding to obtain it analytically. Figure 1 shows magnetization $m\left(h_{a}\right)$ in an increasing applied field $h_{a}$ for an anti-ferromagnetic $\operatorname{RFIM}(J=-1)$, obtained from a simulation of $10^{3}$ spins averaged over $10^{3}$ different realizations of the random field distribution of width $2 \Delta=1$. The magnetization $m\left(h_{a}\right)$ rises from -1 to +1 in three steps. We call these steps as ramp-I $\left(h_{a}=-2|J|-\Delta\right.$ to $\left.h_{a}=-2|J|+\Delta\right) ; \operatorname{ramp-II}\left(h_{a}=-\Delta\right.$ to $\left.h_{a}=+\Delta\right)$;

and ramp-III $\left(h_{a}=2|J|-\Delta\right.$ to $\left.h_{a}=2|J|+\Delta\right)$. The ramps are connected to each other by two plateaus; plateau-I $\left(h_{a}=-2|J|+\Delta\right.$ to $\left.h_{a}=-\Delta\right)$; and plateau-II $\left(h_{a}=+\Delta\right.$ to $\left.h_{a}=2|J|-\Delta\right)$. On the plateaus, the magnetization remains constant even though the applied field continues to increase. Plateaus occur for $\Delta \leq|J|$ (small disorder), and simulations suggest that 
magnetization on the plateaus is independent of $\Delta$. Numerically, the magnetization on the plateaus is approximately $m^{I}=-.135$ on plateau-I, and $m^{I I}=.109$ on plateau-II.

The qualitative shape of $m\left(h_{a}\right)$ is easy to understand. Due to the antiferromagnetic interaction between nearest neighbors, spins with both neighbors down are the easiest to be turned up by an applied field increasing in the up direction. Such spins turn up on ramp-I. Next are the spins with one neighbor up and one down which turn up on ramp-II. Spins with both neighbors up require the largest applied field to turn up, and these turn up on ramp-III. On each ramp, the sequence in which the spins turn up is determined by the distribution of the quenched random field. Spins with large positive quenched field turn up before spins with a lower quenched field. The quenched field lies in the range $-\Delta$ to $+\Delta$. Thus each ramp has a width $2 \Delta$ along the axis of the applied field.

When a spin turns up on ramp-I, its nearest neighbors are placed in a category so that they cannot turn up before ramp-II. Similarly when a spin turns up on ramp-II, its nearest neighbor which is down cannot turn up before ramp-III. This is essentially the reason for the absence of avalanches in the anti-ferromagnetic RFIM. Occasionally on ramp-II and ramp-III, a spin turning up can induce its nearest neighbor which is already up to turn down. We will discuss the situation on ramp-II in detail later, but suffice it to say here that this process too does not cause an avalanche. With 
anti-ferromagnetic interactions, spins turn up one at a time (no Barkhausen noise), and the calculation of $m\left(h_{a}\right)$ becomes essentially a matter of sorting quenched random fields in decreasing order on each ramp. The difficulty arises from the fact that the a posteriori distribution of random fields on spins classified according to the ramp on which they turn up is significantly modified from the uniform distribution given in Equation (1). Indeed, the main object of this paper is to calculate this distribution.

\section{RAMP-I}

Magnetization on ramp-I was determined earlier in reference 5 by exploiting a similarity between this problem and the problem of random sequential adsorption(RSA) [च]. The rate equations of the RSA problem were used to determine $m\left(h_{a}\right)$ on ramp-I, but they could not determine $m\left(h_{a}\right)$ on ramp-II and ramp-III. Here we rederive the result for ramp-I by a different approach which can be extended to ramp-II as well.

We start with a large negative applied field $\left(h_{a}=-\infty\right)$ when all spins are down and increase the applied field slowly. At the start of ramp-I, i.e. at $h_{a}=-2|J|-\Delta$, the spin with the largest positive quenched field becomes unstable and flips up. As the applied field continues to increase, the spin with the next largest quenched field turns up unless it happens to be next to a spin which is already up. In this case the spin which turns up next is the one with the largest quenched field from among the spins with both 
neighbors down. Consider an arbitrary point on ramp-I at an applied field $h_{a}=-2|J|-h$. In the following, the field $\mathrm{h}$ will be used more frequently than $h_{a}$. In general, the field $\mathrm{h}$ will be given by the relation $h=-h_{a} \bmod |2 J|$, so that on each ramp it has the same range as the random field $(-\Delta \leq h \leq \Delta)$. At an applied field $h_{a}=-2|J|-h$ all spins with quenched random field $h_{i}>h$ are relaxed, and a fraction of them are up. The fraction of sites with $h_{i}>h$ is given by

$$
p(h)=\int_{h}^{\Delta} p\left(h_{i}\right) d h_{i}=\frac{\Delta-h}{2 \Delta}
$$

The fraction of spins which are up on ramp-I at $h_{a}=-2|J|-h$ is given by

$$
P_{\uparrow}^{I}=\frac{1}{2}\left[1-e^{-2 p}\right]
$$

Let $P_{\downarrow \downarrow}^{I}$ be the probability (per site) of finding a pair of adjacent down spins on ramp-I at the applied field $h_{a}=-2|J|-h$. It can be calculated as follows. Imagine coloring all sites with $h_{i}>h$ black, and all sites with $h_{i}<h$ white. Consider two adjacent down spins A and B shown in Figure (2). The sites A and B can be both white, both black, or mixed. Given that A is down, it is clear that the state of B can only be influenced by the evolution of the system to the right of B. Similarly, given that B is down, the state of A can only be influenced by the evolution of the system to the left of A. We shall refer to this as the principle of conditional independence [ 8$]$. It requires

$$
P_{\downarrow \downarrow}^{I}=P(A \downarrow \mid B \downarrow) P(B \downarrow \mid A \downarrow)
$$


where $P(A \downarrow \mid B \downarrow)$ is the probability that spin at site $\mathrm{A}$ is down given that spin at $\mathrm{B}$ is down, and $P(B \downarrow \mid A \downarrow)$ is the probability that $\mathrm{B}$ is down given that $\mathrm{A}$ is down. We take up the calculation of $P(B \downarrow \mid A \downarrow)$. If $\mathrm{B}$ is a white site, $P(B \downarrow \mid A \downarrow)=1$ because the white sites have not been relaxed from their initial state. If $\mathrm{B}$ is a black site and the site to the right of $\mathrm{B}$ is a white site then $P(B \downarrow \mid A \downarrow)=0$. In general $P(B \downarrow \mid A \downarrow)$ depends on the length of the string of black sites to the right of B. Suppose B is a black site, and there are (n-1) additional black sites to the right of B. In this case, the probability $P_{B}^{n}$ that $\mathrm{B}$ is down satisfies the following recursion relation,

$$
P_{B}^{n}=\frac{1}{n} P_{B}^{n-2}+\left(1-\frac{1}{n}\right) P_{B}^{n-1}
$$

The rationale for the above recursion relation is as follows. Let the black site farthest from $\mathrm{B}$ on the right be labelled as the n-th site. Any of the $\mathrm{n}$ sites could flip first. The probability that the n-th site flips first is therefore equal to $\frac{1}{n}$. If this happens, (n-1)-th site is prevented from flipping up on ramp-I. The probability that $\mathrm{B}$ is down is now reduced to the probability that the end point of a chain of (n-2) black sites is down i.e. $P_{B}^{n-2}$. This accounts for the first term in equation (8). The probability that n-th site is not the first site to flip up is equal to $\left(1-\frac{1}{n}\right)$. Given this situation, the probability that $\mathrm{B}$ is down is equal to the probability that the end of a string of (n-1) black sites is down. This accounts for the second term in equation (8). We can rewrite the recursion relation (8) as

$$
\left(P_{B}^{n}-P_{B}^{n-1}\right)=-\frac{1}{n}\left[P_{B}^{n-1}-P_{B}^{n-2}\right]
$$


It has the solution,

$$
P_{B}^{n}=\sum_{m=0}^{n} \frac{(-1)^{m}}{m !}
$$

Summing over various possible values of $n$ with appropriate weight, we get

$$
\begin{aligned}
P_{B}= & \sum_{n=0}^{\infty} \sum_{m=0}^{n} \frac{(-1)^{m}}{m !} p^{n}(1-p) \\
& =\sum_{m=0}^{\infty} \frac{(-1)^{m}}{m !}(1-p) \sum_{n=m}^{\infty} p^{n}=\sum_{m=0}^{\infty} \frac{(-p)^{m}}{m !}=e^{-p}
\end{aligned}
$$

Thus,

$$
P_{\downarrow \downarrow}^{I}=e^{-2 p}
$$

Let $P_{\downarrow}^{I}$ be the probability per site of finding a down spin and $P_{\downarrow \uparrow}^{I}$ the probability per site of finding a down spin which is followed by an up spin. Clearly,

$$
P_{\downarrow}^{I}=P_{\downarrow \downarrow}^{I}+P_{\downarrow \uparrow}^{I}=1-P_{\uparrow}^{I}
$$

Keeping in mind that on ramp-I an up spin must be preceeded (as well as followed) by a down spin, we get $P_{\downarrow \uparrow}^{I}=P_{\uparrow}^{I}$. Thus,

$$
P_{\uparrow}^{I}=1-P_{\downarrow \downarrow}^{I}-P_{\uparrow}^{I}
$$

or,

$$
P_{\uparrow}^{I}=\frac{1}{2}\left[1-P_{\downarrow \downarrow}^{I}\right]=\frac{1}{2}\left[1-e^{-2 p}\right]
$$

The magnetization on ramp-I is given by 


$$
m^{I}(h)=2 P_{\uparrow}^{I}(h)-1=-e^{-2 p(h)}
$$

Equation (15) has been superposed on the simulation data for ramp-I shown in Figure 1. The fit between the simulation and theory is so good that the two curves are indistinguishable from each other on the scale of

Figure (1). The exact value of the magnetization on plateau-I is equal to $-\frac{1}{e^{2}}$ which is approximately equal to -.135 .

\section{PLATEAU-I}

Plateau-I contains down spins in singlets and doublets punctuated by up spins. The down spins were relaxed on ramp-I but did not turn up because the then applied field was not strong enough to turn up spins with at least one neighbor up. The singlets have two neighbors up, and the applied field on ramp-II is still not strong enough to turn them up. They have to wait for their turn on ramp-III. On the other hand, each spin in a doublet has one neighbor up and one down. It therefore experiences zero net field from its neighbors. The net field on a doublet spin is simply the sum of the random field $h_{i}$ on its site and the applied field $h_{a}=-h$. It turns up when $h_{a}+h_{i} \geq 0$, or $h_{i} \geq h$. Note that the random field is bound in the range $-\Delta<h_{i}<+\Delta$. Therefore an applied field smaller than $-\Delta$ is sufficiently negative to pin down all doublets. This is the reason for the plateau in the magnetization for applied fields in the range $(-2|J|+\Delta)<h<-\Delta$. In each doublet, the spin with the larger quenched field $h_{i}$ flips up on ramp-II when the applied 
field reaches a value such that $h_{i} \geq h$. The spin with the smaller quenched field then becomes a singlet which does not flip up before ramp-III. Thus, in order to find the form of ramp-II, we need to find the a posteriori distribution of quenched random fields on the doublets.

Consider a doublet on plateau-I as shown in Figure (3). The doublet sites are denoted as 1 and 2, and the quenched random fields on these sites are $h_{1}$ and $h_{2}$. The probability (per site) of finding a doublet on plateau-I is easily obtained from equation (12) by putting $p=1$. It is equal to $\frac{1}{e^{2}}$. We now calculate the probability distribution for $h_{1}$ and $h_{2}$. The distribution of $h_{1}$ and $h_{2}$ will be identical if the evolution of the system on the two sides of the doublet is similar to each other. We assume this to be the case for now, although we shall examine a more general situation later. In order to obtain the desired probability distribution, consider the system on ramp-I when all spins are relaxed upto an arbitrary applied field $-2|J|-h$. At this point, the probability per site for finding a doublet is $P_{\downarrow \downarrow}^{I}=e^{-2 p}$, where $\mathrm{p}$ is fraction of sites with $h_{i}>h$ (black sites) on an infinite lattice.

$$
p(h)=\frac{\Delta-h}{2 \Delta}
$$

It is often easier to think in terms of the fraction p. We therefore introduce similar fractions for the quenched fields given by the following relations.

$$
p\left(h_{i}\right)=\frac{\Delta-h_{i}}{2 \Delta} ;(\mathrm{i}=1,2, \ldots)
$$

Given that site 1 is down on ramp-I, the conditional probability that the adjacent site 2 is also down is equal to $e^{-p}$. Site 2 may be black $\left(h_{2}>h\right.$ or 
equivalently $p_{2}<p$ ) with an a priori probability $\mathrm{p}$, or white with an a priori probability (1-p). If site 2 is white $\left(h_{2}<h\right)$, it must be down because white sites are yet to be relaxed. Thus, the conditional probability that the spin at site 2 is down, and the quenched field at site 2 is larger than $\mathrm{h}$ is given by

$$
\operatorname{Prob}\left(2 \downarrow \mid 1 \downarrow, p_{2}<p\right)=\left[e^{-p}-(1-p)\right] .
$$

The probability that the quenched field $h_{2}$ lies in the range $\mathrm{h}$ and $\mathrm{h}$-dh, or equivalently $p_{2}$ is in the range $\mathrm{p}$ and $\mathrm{p}+\mathrm{dp}$ can be obtained by taking the derivative of the above expression. We obtain,

$$
\operatorname{Prob}\left[p<p_{2}<p+d p\right] d p=\left[1-e^{-p}\right] d p
$$

Similarly,

$$
\operatorname{Prob}\left[p<p_{1}<p+d p\right] d p=\left[1-e^{-p}\right] d p
$$

We now address an issue which is crucial for determining ramp-II correctly. This concerns two adjacent doublets on plateau-I as shown in Figure (4). Let $h_{1}, h_{2}, h_{3}, h_{4}$ and $h_{5}$ denote the quenched fields at sites $1,2,3,4$,and 5 respectively. If $h_{2}>h_{1}$, and $h_{4}>h_{5}$, then spins at sites 2,3 , and 4 will be up at some value of the applied field on ramp-II. When this happens, i.e when a triplet of up spins is created on ramp-II, the central spin at site 3 becomes unstable and it flips down. It stays down till the system reaches ramp-III. In order to take this effect into account, we must know the probability per site of observing two adjacent doublets on plateau-I, and also the distribution of fields at sites 2 and 4 . 
A doublet on plateau-I has an important property. It separates the lattice into two parts (one on each side of the doublet) which have evolved uninfluenced by each other on ramp-I. Thus, we can separate Figure (4) into three parts as enclosed in the dashed boxes. Evolution inside each box has remained shielded from the outside on ramp-I. The evolution in the middle box requires that site 3 flips up before site 2 or site 4 . The probability for this event is equal to $\frac{1}{3}$. Given this event, the probability that spins at sites 1 and 5 remain down all along ramp-I is each equal to $\frac{1}{e}$. Thus the probability per site of observing two adjacent doublets on plateau-I is equal to $\frac{1}{3 e^{2}}$. Note that it is quite different from the square of the probability of finding a single doublet!

The shielding property of the dashed boxes in Figure (4) can also be used to calculate the a posteriori distribution of fields $h_{1}, h_{2}, \ldots, h_{5}$. The distribution of $h_{1}$ and $h_{5}$ is the same as obtained above for a doublet with similar evolution on the two sides (Figure 3).

$$
\operatorname{Prob}\left(p \leq p_{1} \leq p+d p\right) d p=\left[1-e^{-p}\right] d p
$$

and,

$$
\operatorname{Prob}\left(p \leq p_{5} \leq p+d p\right) d p=\left[1-e^{-p}\right] d p
$$

We now turn to the distributions of $h_{2}$, and $h_{4}$. Suppose, in the middle box in Figure (4), the central spin at site 3 is up when all sites in the system with quenched fields $h_{i} \geq h$ (black sites) have been relaxed. This necessarily 
means that $h_{3} \geq h$, i.e. site 3 is a black site, but sites 2 and 4 have other options. Site 3 will be up with probability $\frac{1}{3} p^{3}$ if 2 and 4 are both black, probability $p(1-p)^{2}$ if 2 and 4 are both white, and $(1-p) p^{2}$ if 2 and 4 are mixed. Thus the probability of observing two adjacent doublets with the up spin separating them having field $h_{3} \geq h\left(p_{3} \leq p\right)$ is given by

$$
P_{\downarrow \uparrow \downarrow \downarrow \downarrow}\left(p_{3} \leq p\right)=\left[\frac{1}{3} p^{3}+p(1-p)^{2}+(1-p) p^{2}\right] \frac{1}{e^{2}}
$$

In order to calculate the distribution of $h_{2}$ and $h_{4}$, it is convenient to write the distributions of the smaller and the larger of these two fields separately. Without any loss of generality, we can assume

$$
h_{2}=\min \left(h_{2}, h_{4}\right) \text { and } h_{4}=\max \left(h_{2}, h_{4}\right)
$$

If $h_{2} \geq h$, then we have $h_{3} \geq h_{4} \geq h$ as well. Thus the fraction of adjacent doublets on plateau-I with $h_{2}, h_{3}$, and $h_{4}$ all greater than an arbitrary value $\mathrm{h}$ is given by

$$
P_{\downarrow \downarrow \uparrow \downarrow \downarrow}\left(p_{3} \leq p_{4} \leq p_{2} \leq p\right)=\frac{p^{3}}{3} \frac{1}{e^{2}}
$$

The above equation gives the cummulative fraction of $p_{2} \leq p$ sites. The fraction of sites in the range $p+d p \geq p_{2} \geq p$ can be obtained by differentiating the above expression. We get,

$$
P_{\downarrow \downarrow \uparrow \downarrow \downarrow}\left(p \leq p_{2} \leq p+d p\right)=p^{2} \frac{1}{e^{2}}
$$

The distribution of $h_{4}$ is obtained similarly. We find,

$$
P_{\downarrow \downarrow \uparrow \downarrow \downarrow}\left(p \leq p_{4} \leq p+d p\right)=2 p(1-p) \frac{1}{e^{2}}
$$


Given that we have a pair of adjacent doublets, the probability densities of the quantities $p_{1}, p_{2}, p_{4}$, and $p_{5}$ (each normalised to unity) are given by:

$$
\begin{gathered}
\rho_{1}\left(p_{1}\right)=e\left[1-e^{-p_{1}}\right] \\
\rho_{2}\left(p_{2}\right)=3 p_{2}^{2} \\
\rho_{4}\left(p_{4}\right)=6 p_{4}\left(1-p_{4}\right) \\
\rho_{5}\left(p_{5}\right)=e\left[1-e^{-p_{5}}\right]
\end{gathered}
$$

In the following, we focus on adjacent doublets which create up triplets on ramp-II. These are the objects with $h_{2} \geq h_{1}$ and $h_{4} \geq h_{5}$. At this stage, we can determine the lower and the upper bound on these objects at any point on ramp-II when spins with $h_{i} \geq h$ have been relaxed. Suppose we order the adjacent doublets in the order of increasing $h_{2}$ or increasing $h_{4}$. Note that a sequence in increasing $h_{2}$ does not posses any particular order in $h_{4}$, and vice versa. The lower bound is given by the fraction of objects with $h \geq h_{2} \geq h_{1}$ ( $h_{4}$ being free to have any value in the range $h_{2} \leq h_{4} \leq \Delta$ ). We obtain

$$
\begin{aligned}
& \int_{0}^{p} \rho_{2}\left(p_{2}\right) d p_{2} \int_{p_{2}}^{1} \rho_{1}\left(p_{1}\right) d p_{1} \\
& =\left(6+6 p+3 p^{2}\right) e^{1-p}+(1+e) p^{3}-\frac{3}{4} e p^{4}-6 e \\
& =\left[16-\frac{23}{4} e\right](\text { at } p=1)=.369 \text { (approximately). }
\end{aligned}
$$

Thus a minimum of approximately $37 \%$ adjacent doublets will give rise to (unstable) up triplets on ramp-II. 
Similarly, the fraction of adjacent doublets with $h \geq h_{4} \geq h_{5} \geq-\Delta$ is given by

$$
\begin{aligned}
& \int_{0}^{p} \rho_{4}\left(p_{4}\right) d p_{4} \int_{p_{4}}^{1} \rho_{5}\left(p_{5}\right) d p_{5} \\
& =(1+e) p^{2}(3-2 p)-2 e p^{3}+\frac{3}{2} e p^{4}+6 e-6\left(1+p+p^{2}\right) e^{1-p} \\
& =\left[\frac{13}{2} e-17\right](\text { at } p=1)=.668 \text { (approximately). }
\end{aligned}
$$

This gives the upper bound. No more than approximately $67 \%$ of the adjacent doublets can create (unstable) up triplets on ramp-II.

\section{RAMP-II}

Ramp-II is determined by the combination of two opposite terms. The dominant term is the increase in magnetization due to the decrease in the number of doublets. When a doublet disappears, it adds an extra up spin in the system which increases the magnetization. Occasionally, a disappearing doublet creates a string of three up spins. A triplet of up spins is unstable on ramp-II, and the central spin of the triplet flips down as soon as the triplet is created. This decreases the magnetization. In the following, we calculate the above two terms separately.

Refer to Figure (3) for calculating the first term. The probability that the doublet shown in the Figure (3) disappears when spins with $h_{i} \geq h$ are relaxed is given by

$$
P_{\uparrow \uparrow}^{I I}=\frac{2}{e^{2}} \int_{0}^{p} \rho\left(p_{1}\right) d p_{1} \int_{p_{1}}^{1} \rho\left(p_{2}\right) d p_{2}
$$


The factor $\frac{1}{e^{2}}$ is the probability per site of finding a doublet shown in Figure (3). The factor 2 takes care of the fact that either $h_{1}$ or $h_{2}$ may flip up first. The integrals are written on the assumption that the spin at site 1 flips up first. Together they give the probability that $h_{1} \geq h$, and $h_{2} \leq h_{1}$. Note that when a doublet disappears, a pair of adjacent up spins is created. This is the reason for the choice of the subscript on $P_{\uparrow \uparrow}^{I I}$. The superscript indicates that the probability refers to ramp-II. We obtain,

$$
P_{\uparrow \uparrow}^{I I}=\frac{1}{e^{2}}-\left[\left(1+e^{-1}\right)-\left(p+e^{-p}\right)\right]^{2}
$$

We now caculate the fraction of (unstable) up triplets formed in the system when all spins with $h_{i} \geq h$ have been relaxed on ramp-II. Let us refer to Figure (4). Recall that $h_{2} \leq h_{4}$ in this figure. We want the probability that $h_{2} \geq h_{1}, h_{4} \geq h_{5}$, and $h_{2} \geq h$. This is given by,

$$
P_{\uparrow \uparrow \uparrow}^{I I}=\frac{1}{3 e^{2}} \int_{0}^{p} \rho\left(p_{2}\right) d p_{2} \int_{p_{2}}^{1} \rho\left(p_{1}\right) d p_{1}\left[\frac{\int_{0}^{p_{2}} \tilde{\rho}\left(p_{4}\right) d p_{4} \int_{p_{4}}^{1} \rho\left(p_{5}\right) d p_{5}}{\int_{0}^{p_{2}} \tilde{\rho}\left(p_{4}\right) d p_{4}}\right]
$$

The first factor is the probability per site of finding the object shown in Figure (4). The next two integrals give the probability that $h_{2} \geq h$, and $h_{1} \leq h_{2}$. The quantity in the square brackets is understood as follows: When $h_{2}$ is in the range $\mathrm{h}$ and $\mathrm{h}+\mathrm{dh}, h_{4}$ can be anywhere in the range $h_{2}$ to $\Delta$. Let $\tilde{\rho}\left(h_{4}\right)$ be the density of $h_{4}$ in this range. Clearly,

$$
\rho_{2}\left(h_{2}\right)=\int_{h_{2}}^{\Delta} \tilde{\rho}_{4}\left(h_{4}\right) \frac{d h_{4}}{2 \Delta}
$$

or, 


$$
\rho_{2}\left(p_{2}\right)=\int_{0}^{p_{2}} \tilde{\rho}_{4}\left(p_{4}\right) d p_{4}
$$

Thus,

$$
\tilde{\rho}_{4}\left(p_{2}\right)=\frac{d \rho_{2}\left(p_{2}\right)}{d p_{2}}
$$

or,

$$
\tilde{\rho}_{4}\left(p_{4}\right)=6 p_{4}
$$

We get,

$$
\begin{aligned}
P_{\uparrow \uparrow \uparrow}^{I I}= & \frac{1}{3}\left[\frac{3}{2}+\frac{6}{e}-6\left(1+\frac{1}{e}\right) p+3 p^{2}+\left(1+\frac{1}{e}\right)^{2} p^{3}-\frac{5}{4}\left(1+\frac{1}{e}\right) p^{4}+\frac{2}{5} p^{5}\right. \\
& \left.-\left\{6\left(1+\frac{1}{e}\right)-6 p-3\left(1+\frac{1}{e}\right) p^{2}+2 p^{3}\right\} e^{-p}+\left(\frac{9}{2}+3 p\right) e^{-2 p}\right]
\end{aligned}
$$

We show in Figure (5) a comparison of the above expression with a result from the simulation. As may be expected, the agreement between the simulation and the theory is excellent.

Incidentally, an interesting quantity is the ratio of (unstable) up triplets at the end of ramp-II to the fraction of adjacent doublets at the start of rampII. We noted in the previous section that this ratio must lie approximately in the range .369 to .668 . If there were no correlations between adjacent doublets, this ratio would be equal to $\frac{1}{4}$, because the events $h_{2}>h_{1}$, or $h_{4}>h_{5}$ would occur with probability $\frac{1}{2}$. The exact value of the probability that $h_{4} \geq h_{5} \geq-\Delta$ and $h_{2} \geq h_{1} \geq-\Delta$ is given by

$$
P_{\uparrow \uparrow \uparrow}^{I I}=\frac{1}{3 e^{2}}\left[\frac{11}{2}+\frac{7}{4} e-\frac{27}{20} e^{2}\right]
$$


The quantity in the square brackets is approximately equal to .281. We have checked this result rather carefully numerically, and it is born out by the simulations. Finally, putting the various terms together, the probability of an up spin on ramp-II is given by

$$
P_{\uparrow}^{I I}(p)=P_{\uparrow}^{I}(1)+P_{\uparrow \uparrow}^{I I}(p)-P_{\uparrow \uparrow \uparrow}^{I I}(p)
$$

The magnetization on ramp-II is given by

$$
m^{I I}(p)=2 P_{\uparrow}^{I I}(p)-1
$$

This expression has been superposed on the numerical data for ramp-II shown in Figure (1). The agreement between the numerical data and the theory is extremely good. The exact value of the magnetization on plateauII, and its numerical estimate are given by

$$
m^{I I}(1)=\left[\frac{27}{30}-\frac{7}{6} e^{-1}-\frac{8}{3} e^{-2}\right]=.109 \text { (approximately) }
$$

\section{PLATEAU-II}

Each down spin on plateau-II is a singlet. However, there are three different classes of singlets: the singlets formed on ramp-I; singlets formed on ramp-II by a vanishing doublet; and finally the singlets formed on ramp-II by the unstable central spin of an up triplet flipping down. Each class is characterized by its own a posteriori distribution of the random field. 
Let us denote the three distribution densities by $\rho_{1}^{I I}, \rho_{2}^{I I}$, and $\rho_{3}^{I I}$ respectively. Let $P_{1}^{I I}, P_{2}^{I I}$, and $P_{3}^{I I}$ denote the cummulative populations in each class when spins with $h_{i} \geq h$ have been relaxed on ramp-III. We have,

$$
P_{i}^{I I}(p)=\int_{0}^{p} \rho_{i}^{I I}\left(p_{i}\right) d p_{i}, \mathrm{i}=1,2,3 .
$$

It is useful to think of the singlets in each class as being black $\left(h_{i} \geq h\right)$, or white $\left(h_{i}<h\right)$, where $h_{i}$ is the quenched random field at the singlet site, and $\mathrm{h}$ is an arbitrary reference field. In order to calculate ramp-III, we need only the populations of black singlets in each class given by $P_{i}^{I I}(p)$. If needed, one can obtain the density $\rho_{i}^{I I}(p)$ by differentiating $P_{i}^{I I}(p)$ with respect to $\mathrm{p}$.

The fraction of black singlets created on ramp-I is given by,

$$
P_{1}^{I I}(p)=p-\frac{1}{2}\left[1-e^{-2 p}\right]-\frac{2}{e}\left[e^{-p}-(1-p)\right]
$$

The explanation of the above equation is as follows. Imagine ordering the sites of the lattice in order of decreasing quenched field on the site. When all sites with $h_{i} \geq h$ have been relaxed, the fraction of the relaxed sites is equal to $\mathrm{p}$ (the black sites). This fraction is made of the up sites (the second term on the right), black doublet sites (the last term), and the black singlets. Hence the equation for $P_{1}^{I I}(p)$. The last term is written as follows. In each doublet, there are two sites from which we can choose one. This accounts for the factor 2 . The quantity in the square bracket gives the probability that the chosen site is black, and $\frac{1}{e}$ is the probability that the other site can have any allowed value of the quenched field. 
The fraction of black singlets generated by vanishing doublets on ramp-II is given by,

$$
P_{2}^{I I}(p)=\left[e^{-p}-(1-p)\right]^{2}
$$

The above equation is easily understood. It is the probability that both sites of the doublet are black. If both sites of the doublet are black, the one with higher random field must flip up on ramp-II, leaving us with a singlet on plateau-II which is black.

The fraction of black singlets created by unstable triplets requires the calculation of triplets. We have calculated the fraction of triplets as they are formed on ramp-II. What we need now is a similar but different calculation. The point can be understood with a reference to Figure (4). Recall that $h_{3} \geq$ $h_{4} \geq h_{2}$. On ramp-II, we needed the fraction of triplets with $h_{2} \geq h$, because the formation of triplets is controlled by this threshold. The restoration of the triplets on ramp-III is controlled by the condition $h_{3} \geq h$. In the earlier calculation, sites 2,3 , and 4 were all black sites. In the calculation needed now, only site 3 is black. Sites 2 , and 4 are white, and we want $h_{1} \leq h_{2}$, and $h_{5} \leq h_{4}$. The probability for this event is given by

$$
P_{3}^{I I}(p)=2 \int_{0}^{p} d p_{3} \int_{p_{3}}^{1} d p_{4} \int_{p_{4}}^{1}\left[1-e^{-p_{5}}\right] d p_{5} \int_{p_{4}}^{1} d p_{2} \int_{p_{2}}^{1}\left[1-e^{-p_{1}}\right] d p_{1}
$$

As a check, we note that

$$
2 \int_{p_{3}}^{1} d p_{4} \int_{p_{4}}^{1} d p_{2}=\left(1-p_{3}\right)^{2}
$$


Thus, the double integral gives the probability that sites 2 and 4 are white ( compare with the second term on the right hand side of Equation 21 ). The extra terms in $P_{3}^{I I}(p)$ take into account the requirements $h_{5} \leq h_{4}$, and $h_{1} \leq h_{2}$.

$$
P_{3}^{I I}(p)=2 \int_{0}^{p} d p_{3} \int_{p_{3}}^{1}\left[1+e^{-1}-p_{4}-e^{-p_{4}}\right] d p_{4} \int_{p_{4}}^{1}\left[1+e^{-1}-p_{2}-e^{-p_{2}}\right] d p_{2}
$$

We get,

$$
\begin{aligned}
P_{3}^{I I}(p)= & -\left(1+\frac{2}{e}\right)+\left[\frac{1}{4}+\frac{2}{e}+\frac{4}{e^{2}}\right] p-\frac{1}{2}\left[1+\frac{5}{e}+\frac{4}{e^{2}}\right] p^{2}+\frac{1}{3}\left[\frac{3}{2}+\frac{4}{e}+\frac{1}{e^{2}}\right] p^{3} \\
& -\frac{1}{4}\left[1+\frac{1}{e}\right] p^{4}+\frac{1}{20} p^{5}+\left[1+\frac{2}{e}\right] e^{-p}-\frac{2}{e} p e^{-p}+p^{2} e^{-p}+\frac{1}{2}\left[1-e^{-2 p}\right]
\end{aligned}
$$

As a check we note that,

$$
P_{3}^{I I}(1)=\frac{1}{3 e^{2}}\left[\frac{11}{2}+\frac{7}{4} e-\frac{27}{20} e^{2}\right]
$$

This is the same as given by Equation (38). As one may expect, the number of triplets with $h_{3} \geq h$ is larger than triplets with $h_{2} \geq h$, but the two merge at $h=-\Delta$ i.e. $p=1$.

It is of some interest to calculate the number of triplets with $h_{4} \geq h$ as well, although this quantity is not directly needed in the calculation of ramp-III. Denoting this quantity by $P_{4}^{I I}(p)$, we get

$$
P_{4}^{I I}(p)=2 \int_{0}^{p} p_{4} d p_{4} \int_{p_{4}}^{1}\left[1-e^{-p_{5}}\right] d p_{5} \int_{p_{4}}^{1} d p_{2} \int_{p_{2}}^{1}\left[1-e^{-p_{1}}\right] d p_{1}
$$

Note that either $h_{2}$ or $h_{4}$ could have been the larger of the two fields although we have assumed that $h_{4}$ is the larger one. This accounts for the 
factor 2 on the right hand side. The factor $p_{4}$ comes from integrating $p_{3}$ over the range 0 to $p_{4}$. The other terms are self-explanatory. We get,

$$
\begin{gathered}
P_{4}^{I I}(p)=-\left[\frac{1}{2}+\frac{2}{e}\right]+\frac{1}{2}\left[1+\frac{5}{e}+\frac{4}{e^{2}}\right] p^{2}-\frac{2}{3}\left[\frac{3}{2}+\frac{4}{e}+\frac{1}{e^{2}}\right] p^{3}+\frac{3}{4}\left[1+\frac{1}{e}\right] p^{4} \\
-\frac{1}{5} p^{5}+\left[\left(1+\frac{2}{e}\right)\left(1+p+p^{2}\right)+p^{3}\right] e^{-p}-\left[\frac{1}{2}+p\right] e^{-2 p}
\end{gathered}
$$

It can be checked that $P_{4}^{I I}(1)$ also reduces to the expression in Equation (38) as may be expected. In Figure (5), we have shown the theoretical expressions for $P_{\downarrow \downarrow \downarrow}^{I I}(p), P_{3}^{I I}(p)$, and $P_{4}^{I I}(p)$ along with the results from the simulations for the same quantities. The simulations were performed for a system of $10^{3}$ spins, and averaged over $5 \cdot 10^{3}$ different realizations of the random field distribution. The agreement is excellent as may be expected from an exact analytic result. The agreement between the simulation and the theory also justifies (albeit post facto) the implicit assumption in our analysis that the system is self-averaging. The fact that simulations over a relatively small size of the system $\left(10^{3}\right.$ spins $)$ agree with the exact result is due to the superexponential decay of correlations in this system [7].

\section{RAMP-III}

We are not in a position to write the analytic expression for ramp-III at this stage. In the previous section, we have obtained some ingredients which are necessary for this purpose, but these are not sufficient. If the only process occurring on ramp-III were the gradual decrease in the number of singlets initially present on plateau-II, then we have the required information 
to determine ramp-III. However, additional singlets are created (and later destroyed) on ramp-III. Let us illustrate this with an example. Consider a down spin on plateau-II, say with random field $h_{d}$. Its nearest neighbors are necessarily up because plateau-II contains only singlets. The next nearest neighbors can be up or down. Consider a nearest neighbor, and let $h_{u}$ be the random field on it. The site with field $h_{u}$ may have one or both of its nearest neighbors down. Suppose one of the nearest neighbors of $h_{u}$ is up. Now consider the situation when the singlet just flips up on ramp-III at an applied field $h_{a}$, i.e. $-2|J|+h_{d}+h_{a}=\epsilon$, where $\epsilon \geq 0$. The applied field at this point is $h_{a}=2|J|-h_{d}+\epsilon$. If $h_{u}$ is to remain up after $h_{d}$ has flipped up,

we must have $h_{u}-2|J|+h_{a} \geq 0$, or $h_{u}-h_{d}+\epsilon \geq 0$. This is not guaranteed if $h_{u} \leq h_{d}$. In this case $h_{u}$ will flip down when $h_{d}$ flips up, and later flip up again in a higher applied field. The conditional probability distributions which govern the creation of these new singlets and their restoration on rampIII require further careful investigation. We hope to take up this work in a future article.

\section{CONCLUSION}

We have presented an exact solution of the zero-temperature dynamics of the one-dimensional anti-ferromagnetic random field Ising model in a slowly increasing applied field for a limited range of the applied field. This problem was posed a few years ago, but to the best of our knowledge remained un- 
solved in this period. The solution presented here complements the analytic solution of the non-equilibrium dynamics of the ferromagnetic random field Ising model. The key difference from the ferromagnetic case is the absence of avalanches. A large number of physical systems relax by avalanches, but clearly not all of them. Therefore, models of relaxation without avalanches have to be studied as well. The analysis presented here is a small step in this direction. It also attempts to develop the techniques used in the study of the problem of random sequential adsorption (RSA). The relationship between ramp-I and RSA was noted earlier. Indeed, the RSA rate equations were used to solve the problem of ramp-I. However, the problem of ramp-II and ramp-III could not be solved. Here we have solved the problem of ramp-I by an alternate (although not profoundly different) method which focuses on the a posteriori modification of the random field distribution by the dynamical selection process. This method yields more detailed information on ramp-I than was available earlier, and allows us to get an exact expression for ramp-II. We hope that the method employed here will enable us to solve the problem of ramp-III in the near future, and it may be useful in other related problems as well.

We thank D Dhar for useful discussions during a visit to NEHU. 


\section{REFERENCES}

[1] J P Sethna, K Dahmen, S Kartha, J A Krumhansl, B W Roberts, and J D Shore, Phys Rev Lett 70, 3347 (1993).

[2] D Dhar, P Shukla, and J P Sethna, J Phys A: Math. Gen. 30,5259 (1997).

[3] P Shukla, Physica A 233, 235 (1996).

[4] J Kisker,H Rieger, and H Schreckenberg, J Phys A: Math. Gen. 27, L853 (1994). This paper discusses the non-equilibrium dynamics of a nonrandom, one-dimensional Ising model, with three-spin interactions, at low temperatures. It is qualitatively similar to the dynamics of the random field Ising model at zero-temperature.

[5] P Shukla, Physica A 233, 242 (1996).

[6] S Sabhapandit, P Shukla, and D Dhar, submitted for publication.

[7] J W Evans, Rev Mod Phys 65, 1281 (1993).

[8] L G Mityushin, Prob Peredachi Inf 9, 81 (1973). Also see reference (7) for a detailed discussion of the screening property of this class of problems. 


\section{Figure Captions}

Figure 1:

Magnetization $m\left(h_{a}\right)$ in an applied field $h_{a}$ for an anti-ferromagnetic $\operatorname{RFIM}(J=-1)$, obtained from a simulation of $10^{3}$ spins averaged over $10^{3}$ different realizations of the random field distribution of width $2 \Delta=1$. The solid line shows the magnetization in increasing field. The broken line shows the return half of the hysteresis loop. The data in increasing field is separated into five parts along the applied field: Ramp-I $\left(h_{a}=-2|J|-\Delta\right.$ to $\left.h_{a}=-2|J|+\Delta\right)$;

Plateau-I $\left(h_{a}=-2|J|+\Delta\right.$ to $\left.h_{a}=-\Delta\right)$; Ramp-II $\left(h_{a}=-\Delta\right.$ to $\left.h_{a}=+\Delta\right)$; Plateau-II $\left(h_{a}=+\Delta\right.$ to $\left.h_{a}=2|J|-\Delta\right)$; and Ramp-III $\left(h_{a}=2|J|-\Delta\right.$ to $\left.h_{a}=2|J|+\Delta\right)$. Theoretical expressions obtained in the paper have been superposed on Ramp-I, Plateau-I, Ramp-II, and Plateau-II in increasing field.

Figure 2:

Spins on Ramp-I in an applied field $-2|J|-h$. Filled circles show sites with quenched field $h_{i}>h$. The probability per site of a doublet (two adjacent down spins) such as $\mathrm{AB}$ is equal to $e^{-2 p}$, where $\mathrm{p}$ is 
the fraction of filled circles on the infinite lattice.

Figure 3:

A doublet on Plateau-I: $h_{1}$ and $h_{2}$ are the quenched random fields on the doublet sites 1 and 2 respectively.

Figure 4:

Two adjacent doublets on Plateau-I: Each doublet separates the lattice into two parts whose evolution histories on Ramp-I are independent of each other. Evolutions inside each dashed box is shielded from outside. The probability that spin at site 3 flips up on Ramp-I is therefore equal to $\frac{1}{3}$. Given this, the probability that the spins at sites 1 and 5 remain down all along Ramp-I is equal to $\frac{1}{e}$ each. The shielding property of the boxes can also be used to determine a posteriori distribution of random fields $h_{1}, h_{2}, h_{3}, h_{4}$, and $h_{5}$.

Figure 5:

Unstable up triplets on Ramp-II with $h_{2} \geq h$ (lower curve); $h_{4} \geq h$ (middle curve); and $h_{3} \geq h$ (upper curve). Refer to Figure 4 for $h_{2}$, $h_{4}$, and $h_{3}$. The simulation data is shown by lines, and the symbols on each line show the corresponding theoretical prediction. 


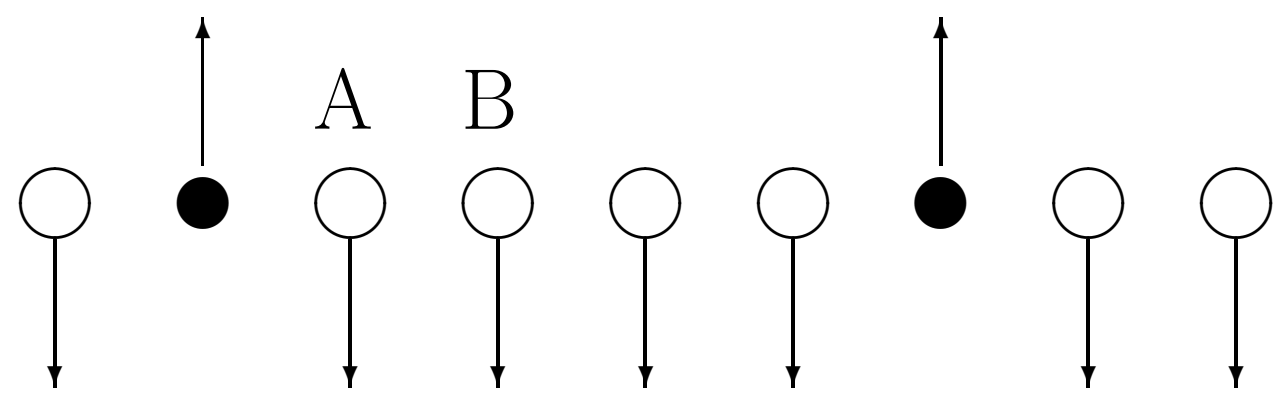

Figure 2
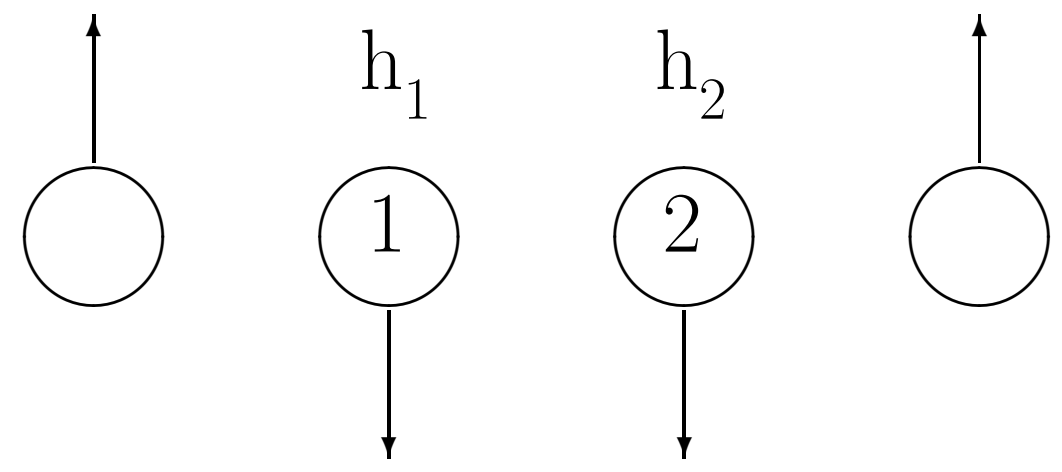

Figure 3 


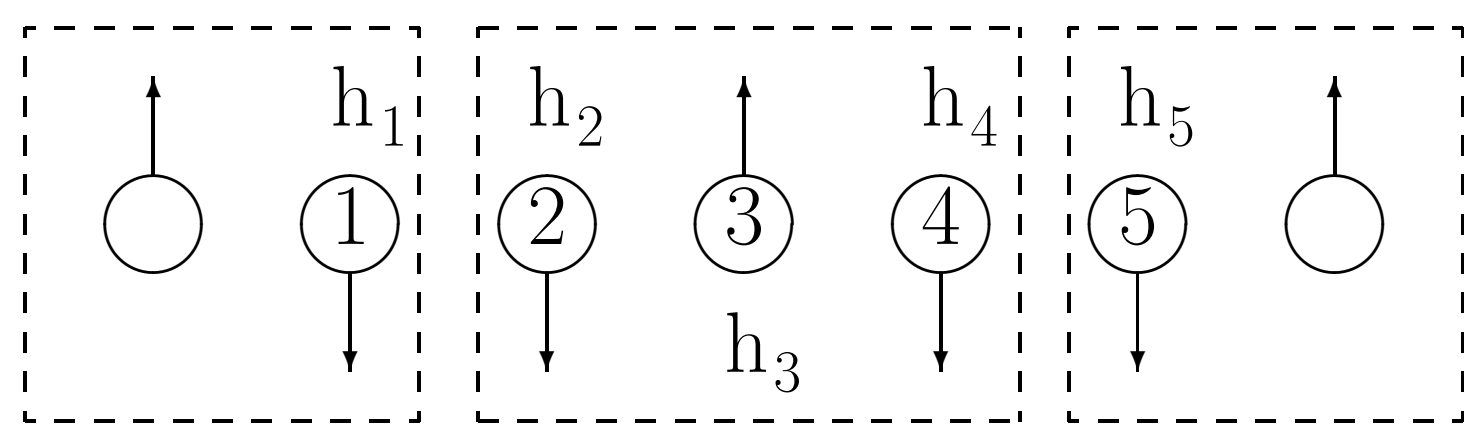

Figure 4 
Figure 1

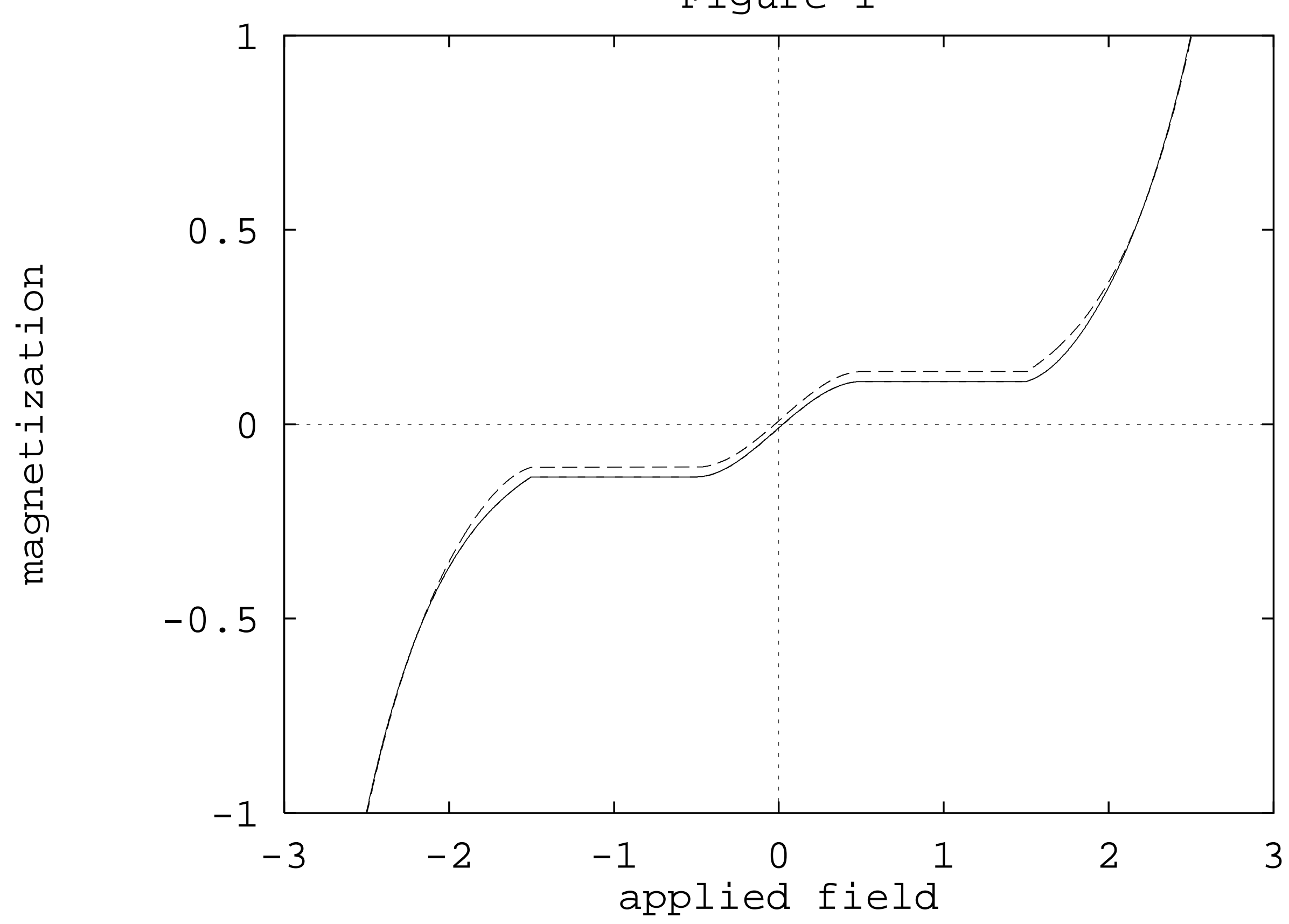


Figure 5

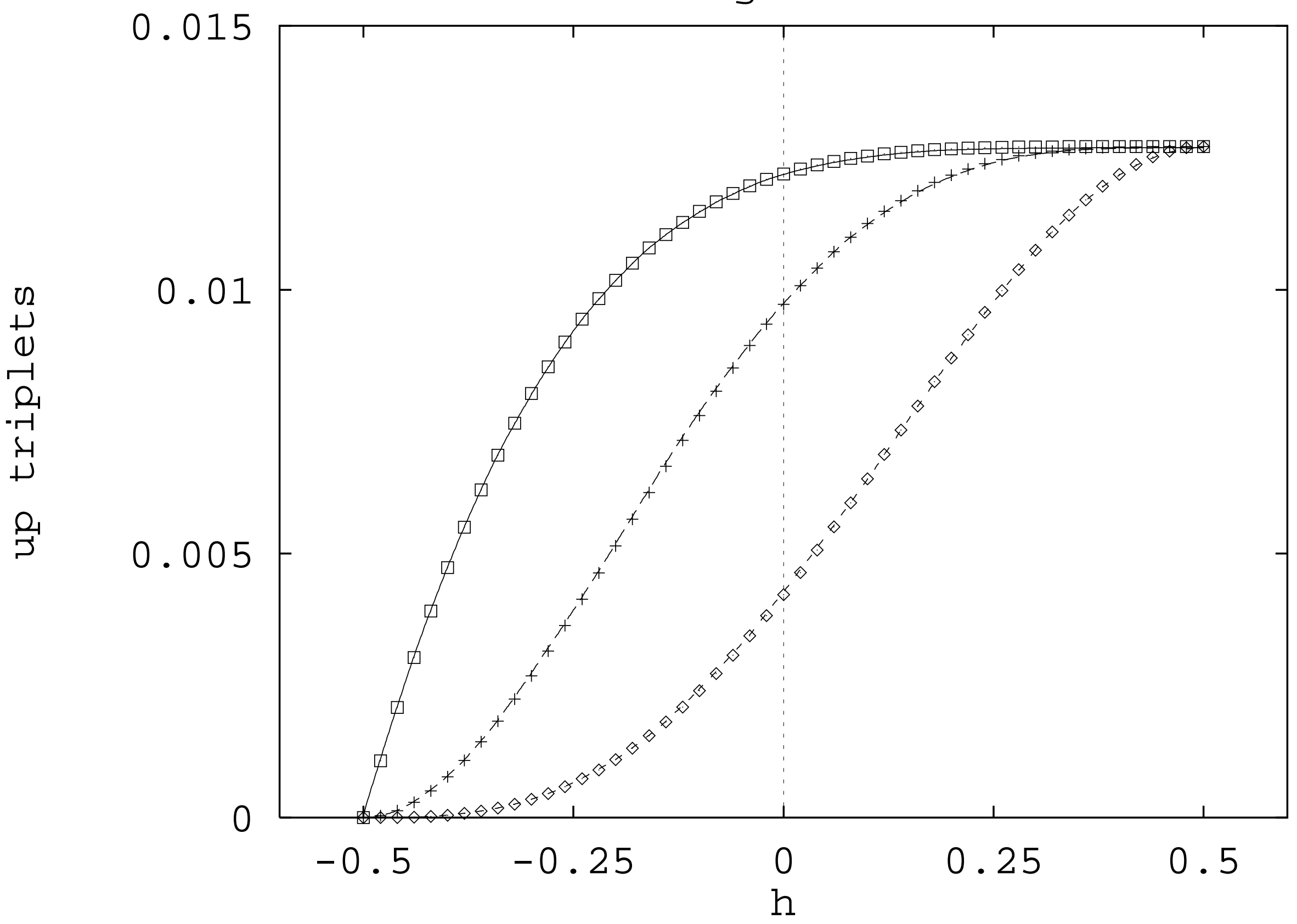

